BULL. AUSTRAL. MATH. SOC.

VOL. 33 (1986), 253-260.

\title{
A NOTE ON THE COEFFICIENTS OF
}

\section{MIXED NORMMED SPACES}

\author{
E,G. KWON
}

For $0<p, q<\infty, \alpha>-1, A^{p, q, \alpha}$ denotes the space of all holomorphic functions in the unit disc satisfying

$$
|| f||_{p, q, \alpha}^{p}=\int_{0}^{1} M_{q}(r, f)^{p}(1-r)^{\alpha} d r<\infty,
$$

where

$$
M_{q}(r, f)^{q}=\frac{1}{2 \pi} s_{0}^{2 \pi}\left|f\left(r e l^{\theta}\right)\right|^{q} d_{\theta} .
$$

In this paper, we find a sufficient condition for the multipliers from $A^{p, q, \alpha}$ into $\ell^{s}, 1 \leq s \leq \infty, 1 \leq q \leq 2$, which interpolates the results of Patrick Ahern and Miroljub Jevtic. As a corollary, we can calculate

$$
\left(A^{p, q, \alpha}, \ell^{s}\right)
$$

for $q^{\prime} \leq s \leq \infty, 1 / q+1 / q^{\prime}=1$. Also, we can find a sharp coefficient condition for $H^{p}$ functions.

\section{Introduction.}

$H(U)$ denotes the space of all holomorphic functions in the unit disc $U$. For a function $f(z) \varepsilon H(U)$ and for $\alpha>-1,0<p, q<\infty$,

Received 2 June 1985.

Copyright Clearance Centre, Inc. Serial-fee code: 0004-9727/86 $\$ A 2.00+0.00$. 
we let

$$
\begin{gathered}
M_{p}(r, f)^{p}=1 / 2 \pi s_{0}^{2 \pi}\left|f\left(r e^{l \theta}\right)\right|^{p} d \theta, \\
|| f||_{p}=\sup _{r} M_{p}(r, f)
\end{gathered}
$$

and

$$
|| f||_{p, q, \alpha}^{p}=\int_{0}^{1} M_{q}(r, f)^{p}(1-r)^{\alpha} d r .
$$

The spaces $H^{p}(U)$ and $A^{p, q, \alpha}(U)$ are defined to be

$$
\begin{aligned}
& H^{p}(U)=\left\{f \in H(U) ; \quad \mid f \|_{p}<\infty\right\} \\
& A^{p, q, \alpha}=\left\{f \in H(U) ;|| f \|_{p, q, \alpha}<\infty\right\} .
\end{aligned}
$$

These spaces form Banach spaces or Frechet spaces. We refer to [3], [1] for properties of these spaces.

Let $A, B$ be two vector spaces of complex sequences. A sequence $\lambda=\left\{\lambda_{n}\right\}$ is said to be a multiplier from $A$ into $B$ if $\left\{\lambda_{n} a_{n}\right\} \in B$ for any $\left\{a_{n}\right\} \in A$. The space of all such multipliers is denoted by $(A, B)$. We want to calculate multipliers from $H^{p}$ or $A^{p, q, \alpha}$ into $\ell(s, t)$, the space defined below.

DEFINITION. For $1 \leq s, t \leq \infty$, we denote by $\ell(s, t)$ the set of those sequences $\left\{a_{k}\right\}_{0}^{\infty}$ for which

$$
\left\{\left(\sum_{k \in I_{n}}\left|a_{k}\right|^{s}\right)^{1 / s}\right\}_{n=0}^{\infty} \varepsilon e^{t}(s<\infty)
$$

and

$$
\left\{\sup _{k \in I_{n}}\left|a_{k}\right|\right\} \cos _{n=0}^{\infty} \varepsilon e^{t}(s=\infty),
$$

where $I_{n}=\left\{k ; 2^{n}<k \leq 2^{n+1}\right\}(n=1,2, \ldots)$ and $I_{0}=\{0\}$.

The $\ell(s, t)$ form normed spaces. For dual spaces and multipliers

between these spaces we refer to [5]. We follow Anderson and Shields [2]

for notation and many results. Let $A$ be a sequence space. $A^{\alpha}$ is

defined to be the space of sequences $\left\{a_{n}\right\}$ for which 


$$
\lim _{r \rightarrow 1-} \sum a_{n} r^{n}
$$

exists and $A^{k}$ is defined to be $\left(A, \ell^{1}\right) . s(A)$ is defined to be the largest subspace of $A$ having the property that if $\left\{a_{n}\right\} \varepsilon s(A)$ and $\left|b_{n}\right| \leq\left|a_{n}\right|$ then $\left\{b_{n}\right\} \in s(A)$. Similarly $S(A)$ is defined to be the smallest superspace having this property. If $s(A)=S(A)$, we call the space $A$ solid. Of course, the $\ell(s, t)$ are solid. It is known [2] that for a solid space $X$ with $X^{k k}=X$

$$
(A, X)=(S(A), X)=\left(A^{k k}, X\right)=\left(s\left(A^{a}\right)^{a}, X\right) .
$$

Note that $f \in H(U)$ can be identified as a sequence $\left\{a_{n}\right\}$ if $f(z)=\sum_{0}^{\infty} a_{n} z^{n}$. We denote $\left\{(n+1)^{-p} a_{n}\right\}$ by $I_{f}^{p}$ and $\left\{(n+1)^{p} a_{n}\right\}$ by $I^{-p} f$, the fractional integral and the fractional derivative of $f$ of order $p$. Also, for a space $S \subset H(U)$ we denote $\left\{I^{p} f ; f \varepsilon S\right\}$ by $I^{p} S$ and for two sequence spaces $A, B$ we denote $\left\{\left\{a_{n} b_{n}\right\} ;\left\{a_{n}\right\} \in A,\left\{b_{n}\right\} \in B\right\}$ by $A * B$. Note that

$$
\left\{(n+1)^{-p}\right\} \varepsilon \&(1 / p, \infty)
$$

so that

$$
I_{A} P \ell(1 / p, \infty) * A
$$

Throughout this paper, $1 / p+1 / p^{\prime}=1(1 \leq p \leq \infty)$ and $c(p, q, \ldots)$ denotes a positive constant depending only on $p, q, \ldots$, but its size may vary under the same notation.

\section{Results}

Ahern and Jevtić[1] have calculated multipliers from $A^{p, q, \alpha}$ into $\ell^{S}$ in the case $0<p<\infty, q=1,2$. They prove that if $r=\max (p, 1)$ then

$$
\begin{aligned}
& \left(s \left(A^{p, 1, \alpha, a, a}=\left\{\left[\lambda_{n}\right\} ;\left\{(k+1) \frac{\alpha+1}{p}_{\lambda_{k}}\right\} \in \ell(\infty, r)\right\}\right.\right. \\
& \left(s\left(A^{p, q, \alpha, a, a}=\left\{\left\{\lambda_{k}\right\} ;\{(k+1))^{-\frac{\alpha+1}{p}} \lambda_{k}\right\} \in \ell(2, r)\right\} \quad(q \geq 2)\right.
\end{aligned}
$$


256

E. G. Kwan

(Indeed, they proved these for $1<p<\infty$ and remarked on the case $0<p \leq 1$. See [1] Remarks.) Noting that

$$
A^{p, q, \alpha} \subset\left(s\left(A^{p, q, \alpha}\right)^{a}\right)^{a}
$$

it is natural to conjecture the following:

THEOREM 1. For $1 \leq q \leq 2, A^{p, q, \alpha} \subset I^{-(\alpha+1) / p} \ell\left(q^{\prime}, \max (p, 1)\right)$, where $1 / q+1 / q^{\prime}=1$.

Proof. Note that $\lambda=\left\{\lambda_{n}\right\} \in I^{-(\alpha+1) / p} \ell\left(q^{\prime}, p\right)$ if and only if

$$
\sum_{0}^{\infty}\left(k \sum_{\varepsilon} I_{n}\left|(k+1)^{-(\alpha+1) / P} \lambda_{k}\right|^{q^{\prime}}\right)^{p / q^{\prime}<\infty}
$$

Let $f(z)=\sum_{0}^{\infty} a_{n} z^{n} \in H(U)$. Then

$$
\begin{aligned}
& \left.\sum \sum_{I_{n}} \mid(k+1)^{-(\alpha+1) / p} a_{k} q^{\prime}\right)^{p / q^{\prime}} \\
\leq & \sum 2^{-n(\alpha+1)}\left(\sum_{I_{n}}\left|a_{k}\right|^{\left.q^{\prime}\right)^{p / q^{\prime}} .}\right.
\end{aligned}
$$

Applying the result of Mateljević and Pavlović [6], this term is dominated by

$$
C(p, q, \alpha) \int_{0}^{1}\left(\sum_{0}^{\infty}\left|a_{k}\right|^{q^{\prime}} r^{k}\right)^{p / q^{\prime}}(1-r)^{\alpha} d r .
$$

since $\sum_{0}^{\infty} a_{k} r^{k / q^{\prime}} z^{k}=f\left(r^{1 / q^{\prime}} z\right)$, the Hausdorff-Young theorem $[3$.

Theorem 6.1] gives

$$
\begin{aligned}
\left(\sum_{0}^{\infty}\left|a_{k}\right|^{\left.q^{\prime} r^{k}\right)^{1 / q^{\prime}}}\right. & =\left(\sum_{0}^{\infty} \mid a_{k} r^{k / q^{\prime} \mid q^{\prime}}\right)^{1 / q^{\prime}} \\
& \leq \|\left. f\left(r^{1 / q^{\prime}} z\right)\right|_{q}
\end{aligned}
$$

for $1<q<2$. On the other hand, if we let $f_{p} 1 / q^{\prime}(z)=f\left(r^{1 / q^{\prime}} z\right)$, simple calculations give

$$
\begin{aligned}
& \left.\int_{0}^{1}|| f\left(r^{1 / q^{\prime}} z\right)\right|_{q} ^{p}(1-r)^{\alpha} d r \\
= & \int_{0}^{1} \sup _{p} M_{q}\left(\rho, f r_{r}^{1 / q^{\prime}}\right)^{p}(1-r)^{\alpha} d r \\
= & \int_{0}^{1} M_{q}\left(r^{1 / q^{\prime}}, f\right)^{p}(1-r)^{\alpha} d r
\end{aligned}
$$

https://doi.org/10.1017/S0004972700003129 Published online by Cambridge University Press 


$$
\begin{aligned}
& =q^{\prime} \int_{0}^{1} M_{q}(\rho, f)^{p}\left(1-\rho^{q^{\prime}}\right)^{\alpha} \rho^{q^{\prime}-1} d \rho \\
& \leq\left(q^{\prime}\right)^{1+\alpha} \int_{0}^{1} M_{q}(\rho, f)^{p}(1-\rho)^{\alpha} d \rho,
\end{aligned}
$$

where we used the fact that $\left(1-\rho^{q^{\prime}}\right) \leq q^{\prime}(1-\rho)$ in the last inequality. Thus we have

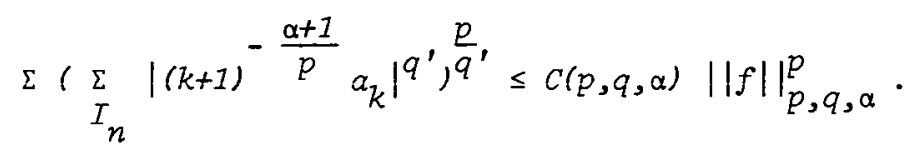

This process can also be applied when $p \leq 1$ by the duality method aforementioned ([1] Remarks). The proof is now complete.

COROLLARY 1. If $0<p \leq 2$ and $p<q, 1 \leq q \leq \infty$, then

$$
H^{p} \subset I^{1 / q-1 / p} \ell\left(q^{\prime}, r\right)
$$

where $r=\max (p, 1)$. That is, $f=\left\{a_{n}\right\} \varepsilon H^{p}$, then

$$
\sum_{n}\left(\sum_{I_{n}}\left|(k+1)^{\frac{1}{q}-\frac{1}{p}} a_{k}\right|^{q^{\prime}, \frac{r}{q},}<\infty,\right.
$$

with the obvious understanding when $q=1$ or $q=\infty$.

Proof. First we note that it suffices to prove (1) for $1 / q-1 / p$ small. Indeed, if $\left\{a_{k}\right\} \in I^{1 / q_{1}-1 / p} \ell\left(q_{1}^{\prime}, r\right)$ and $1 \leq q_{1} \leq q_{2} \infty^{\infty}$, then

$$
\left\{(k+1)^{1 / q_{1}-1 / p} a_{k}\right\} \in \ell\left(q_{1}^{\prime} \cdot r\right)
$$

Since

$$
(k+1)^{1 / q_{2}-1 / q_{1}} \in \ell\left(\frac{1}{1 / q_{1}-1 / q_{2}}, \infty\right)=\left(\ell\left(q_{1}^{\prime}, r\right),\left(q_{2}^{\prime}, r\right)\right),
$$

we have

$$
\begin{aligned}
\left\{(k+1)^{1 / q_{2}-1 / p} a_{k}\right\} & =\left\{(k+1)^{1 / q_{2}-1 / q_{1}}(k+1)^{1 / q_{1}-1 / p} a_{k}\right\} \\
& \in \ell\left(\frac{1}{1 / q_{1}-1 / q_{2}}, \infty\right) * \ell\left(q_{1}^{\prime}, r\right) \\
& \subset \ell\left(q_{2}^{\prime}, r\right) .
\end{aligned}
$$


Thus, we have proven that

$$
I^{1 / q_{1}-1 / p} \ell\left(q_{1}^{\prime}, r\right) \subset I^{1 / q_{2}-1 / p} \ell\left(q_{2}^{\prime}, r\right)
$$

in the case that $1 \leq q_{1}<q_{2} \leq \infty$.

Now, if $0<p<2$, the Hardy-Littlewood theorem [3. Theorem 5.11] and Theorem 1 gives

$$
H^{p} \subset A^{p, q,-p / q} \subset I^{1 / q-1 / p} \ell\left(q^{\prime}, r\right)
$$

by taking $q<2$. Finally, the remaining case when $p=2$ is easy

$$
\begin{aligned}
I^{1 / 2-1 / q} H^{2} & \subset \ell\left(\frac{1}{1 / 2-1 / q^{\prime}}, \infty\right) * \ell^{2} \\
& \subset \ell\left(q^{\prime}, 2\right),
\end{aligned}
$$

because

$$
\ell\left(\frac{1}{1 / 2-1 / q^{\prime}}, \infty\right)=\left(l^{2}, \ell\left(q^{\prime}, 2\right)\right) .
$$

Hence

$$
H \subset I^{1 / q-1 / 2} \ell\left(q^{\prime}, 2\right) \text {. }
$$

Since $H^{P^{\prime}}=\left(H^{p}\right)^{k}=\left(H^{p}, \ell^{1}\right)$ if $1<p \leq 2$, we have the dual form of (1) as follows.

\section{COROLLARY 2.}

$$
\begin{aligned}
& H^{p}>I^{1 / q-1 / p} \ell\left(q^{\prime}, p\right), \quad 2 \leq p<\infty, \quad 1 \leq q<p . \\
& B M O A(U)>I^{1 / q} \ell\left(q^{\prime}, \infty\right), \quad 1 \leq q<\infty
\end{aligned}
$$

[where $B M O A(U)$ is the space of analytic functions on $U$ having bounded mean oscillation].

Remarks. 1. If we set $q=2$ in (1) and (2) then we have $H^{p} \subset D^{p}$ for $p<2$ and $H^{p} \supset D^{p}$ for $p>2$. (see [j], [4] for $D^{p}$ ). Thus Corollary 1 and Corollary 2 are stronger than [ 3 . Theorems 6.2, 6.3] and [4. Theorems $\mathrm{C}, \mathrm{D}]$.

2. The limiting case of (1) namely $H^{p} \subset \ell\left(p^{\prime}, p\right)(1 \leq p<2)$ is not true (thus neither is the limiting case of (2)): If we suppose $H^{p} \subset \ell\left(p^{\prime}, r\right)$ then $S\left(H^{p}\right) \subset \ell\left(p^{\prime}, r\right)$, so 


$$
\ell^{\infty} \subset\left(S\left(H^{p}\right), \ell\left(p^{\prime}, r\right)\right)=\left(H^{p}, \ell\left(p^{\prime}, r\right)\right) .
$$

But $H^{2} \subset H^{p} \subset \ell\left(p^{\prime}, 2\right)[5]$ gives

$$
\left(H^{2}, \ell\left(p^{\prime}, r\right)\right)>\left(H^{p}, \ell\left(p^{\prime}, r\right)\right)>\left(\ell\left(p^{\prime}, 2\right), \ell\left(p^{\prime}, r\right)\right) .
$$

Hence

$$
\left(H^{p}, \ell\left(p^{\prime}, r\right)\right)= \begin{cases}\ell(\infty, 2 r / \bar{Z}-r) & \text { if } 1 \leq \gamma^{\prime} \leq 2, \\ \ell^{\infty} & \text { if } 2 \leq r \leq \infty .\end{cases}
$$

Therefore $\ell^{\infty} \subset\left(H^{p}, \ell\left(p^{\prime}, r\right)\right)$ only if $2 \leq r \leq \infty$. That is there is no ordered pair $\left(p^{\prime}, r\right) r<2$ satisfying $H^{p} \subset \ell\left(p^{\prime}, r\right)$. We can say (1) is sharp in this sense. Also, if $0<p<1$, then from (1) we have $H^{p} \subset I^{1-1 / p} \ell(\infty, 1)$, but $I^{1-1 / p} \ell(\infty, 1)$ is $\left(H^{p}\right)^{k k}$ ([3. Theorem 6.6] Recall that $\left.A^{k}=\left(A, \ell^{1}\right)\right)$.

COROLLARY 3. Let $1 \leq q \leq 2$ and $r=\max (p, 1)$. Then

$$
\begin{aligned}
& \left(A^{p, q, \alpha}, \ell^{s}\right)=I^{\alpha+1 / p}\left(\ell\left(q^{\prime}, r\right), \ell^{s}\right), 1 \leq s \leq \infty, \\
& \left(A^{p, q, \alpha}, \ell^{s}\right)=I^{\alpha+1 / p} \ell(\infty, t), \quad q^{\prime} \leq s \leq \infty .
\end{aligned}
$$

where $t=s p / p-s$ if $s \leq p$

and $\quad t=\infty$ if $s \geq p$.

Proof. (4) is obvious from Theorem 1. We prove (5). Jensen's inequality gives that $M_{q}(\rho, f) \leq M_{2}(\rho, f)$ so that $A^{p, 2, \alpha} \subset A^{p, q, \alpha}$, which in turn gives

$$
\begin{aligned}
\left(A^{p, q, \alpha}, \ell^{s}\right) \subset\left(A^{p, 2, \alpha}, \ell^{s}\right) & =\left(I^{-(\alpha+1) / p} \ell(2, r), \ell^{s}\right) \\
& =I^{\alpha+1 / p}\left(\ell(2, r), l^{s}\right) .
\end{aligned}
$$

Combining this with (4) gives (5).

\section{References}

[1] P. Ahern and M. Jevtic', "Duality and multpliers for mixed norm spaces", Michigan Math. J. 30 (1983), 53-63.

[2] J. M. Anderson and A. L. Shields, "Coefficient multipliers of Bloch functions", Trans. Amer. Math. Soc. 224 (1976), 255-265. 
[3] P. L. Duren, Theory of $H^{p}$ spaces, (Academic Press, New York, 1970).

[4] F. Holland and J. B. Twomey, "Conditions for membership of Hardy spaces", Aspect of contemporary analysis, (Academic Press, London 1980), 425-433.

[5] C. N. Kellog, "An extension of the Hausdorff-Young theorem", Michigan Math. J. 18 (1971), 121-127.

[6] M. Mateljevid and M. Pavlovic, " $I$-behaviour of power series with positive coefficients and Hardy spaces", Proc. Amer. Math. Soc. 87 (1983), 309-316.

Department of Mathematics Education

Andong National University

Andong Kyungpook 660

Korea. 